\title{
PAVEMENT INSTRUMENTATION FOR CONDITION ASSESSMENT USING EFFICIENT SENSING SOLUTIONS
}

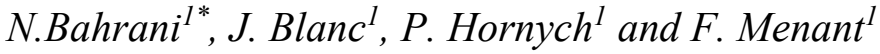 \\ ${ }^{1}$ IFSTTAR, centre de Nantes, Bouguenais CEDEX, France \\ * Corresponding author
}

\begin{abstract}
The practices used for assessing pavement deflections mostly comprise traditional methods under static load like Benkelman beam or Falling Weight Deflectometer. While these techniques are widely used, they have the limitation of requiring the use of a measurement vehicle, which limits the measurement frequency (typically, test are made once every one or two years), and requires the closure of the highway lane. An alternative solution consists in measuring the deflection using sensors embedded in the pavement, in order to measure and record the pavement response continuously, under real traffic. These sensors can be connected to a wireless data acquisition system, enabling continuous and remote monitoring. The purpose of this study is to determine efficient and accurate solutions for measuring the pavement deflections using two types of geophones. Their respective measurements are converted into vertical displacements to find the deflection basin induced. For this purpose, laboratory tests of these geophones have first been performed, using a vibrating table to verify their characteristics and to determine their response to signals simulating the deflection of a pavement under vehicle loading. Different methods of filtering, and processing of the sensor signals have been proposed, in order to obtain accurate measurements of the vertical displacements. The purpose of the test carried out with these geophones also signifies that these sensors are deemed fit for the continuous monitoring and in future could be deployed at road infrastructure with a wireless data acquisition system. Ultimately, the aim is to develop a fully automated method for gathering and processing the sensor measurements, to quantify the performance of the pavement.
\end{abstract}

\section{Introduction}

In pavement engineering, instrumentation of the pavements using sensor is one of the most successful and effective methods to monitor the pavement conditions. It can be used to measure stress, deformation and environmental conditions (temperature, moisture), depending on the materials and the structure of the pavement. Some of these conditions like moisture infiltration, cracking and aging of asphalt pavements affect their life duration making it essential to monitor the pavements. This study concentrates on the measurement of vertical displacements, under vehicle loads, determining deflection basins of pavement and thus could be used to backcalculate its layer moduli. Monitoring of deflections with the course of time can be helpful to follow evolution of pavement layer moduli, and thus to detect the change in layer properties, indicating material damage. It is therefore important to have an accurate detection of the pavement deflections.

In this work laboratory study is carried out for the response of geophones and signals simulating pavement deflections under vehicle loading. This study focuses on improving the treatment of the geophone signals to obtain accurate deflection measurements. The results are validated by comparing the geophone measurements with measurements made with a reference displacement sensor. The objective of the test programme is to develop a procedure for accurate measurement of vertical displacements (deflections) of pavements using geophones. These sensor, installed in vertical position in the pavement, measure the vertical velocity produced by the passing of a wheel load on the pavement. This velocity is integrated and converted into a vertical displacement, representing the pavement deflection. One of the difficulties with these sensors is their specific response function, which depends on signal frequency. Therefore, the geophone measurements need to be treated and corrected in order to filter the measured noise and correct the response. The proposed approach used for testing the transducers to determine the displacements from the geophone measurements is described below:

- Section 2 describes the previous work on the instrumentation of pavements and vertical displacements

- Section 3 deals with the selection of appropriate geophones for the measurement of displacements in pavements

- In section 4 pavement modelling calculations are done to determine typical deflection signals (under five axle truck load), using the linear elastic pavement 
modelling software Alizé. These signals will be used to simulate truck loading in the laboratory tests.

- Section 5 discusses the measured responses of geophones in the laboratory: The test consist of placing the geophones on a vibrating table, and submitting them to the previously calculated deflection signals, corresponding to different amplitudes and velocities.

- Section 6 elaborates the method to filter, correct and integrate the geophone signals, to determine the deflections.

- Finally in section 7, the results have been presented comparing the deflections obtained with the geophones with the reference displacement measurements made with the laser sensor, and adjusting the processing of the geophone signals, until a good match is obtained.

\section{Related work}

The concept of instrumenting the pavement with sensors, to measure the pavement deflections has been wide research topic in pavement Engineering. Many studies (Lajnef N et al ,2011 , Arraigada M et al 2009, Levenberg E, 2012, Alavi et al 2016) have emphasized on the importance of instrumentation and used different approaches like using Piezo electric sensors, accelerometers and geophones to instrument the pavement. The goal is to find the pavement deflections and properties of the structure and to examine the factors effecting the pavement. In (Tholen et al 1985) different methods of measuring the deflections were described, specifically with Falling weight deflectometer (FWD) to monitor pavement behaviour performance with respect to the layer properties. These methods are then compared, evaluated and indicated the need of more field tests.

The work which specifically focuses on geophones is defined in (Liu. $\mathrm{P}$ et al 2017) where the response of asphalt pavement is measured through geophones which are then used to back calculate the pavement properties and describe the deteriorations under moving loads. This work indicates that the geophones were able to recode the small vibration during the moving loads and analyse the evolution in the moduli of the pavement. In (Clark R et al 1991) the goal is to understand the geophones used in the FWD measurements and evaluate their accuracy. This work focuses on the theoretical as well as the calibration tests done on these transducers and describing the pavement displacement. In (Ngoc Son et al 2018) geophones are used to measure pavement deflections and to monitor evolution of pavement layer properties with respect to traffic and environmental conditions. Also focusing on the need to have better processing techniques to get accurate data from geophones.

There is still a need to work on the information retrieved from these sensors and improving the results in order to maintain the accuracy, which is the main objective of this study. In this work, we have focused on geophones, which are lightweight and relatively low cost sensors, which are used to measure displacement velocities. They are very sensitive, which makes them suitable to measure very small ground displacements. Most geophones are based on the principle of a magnetic mass suspended on a spring, moving within a wire coil (Nazarian et al 1989). They deliver an electrical signal which is proportional to the vertical displacement velocity. The response of geophones is non linear, and is characterised by an attenuation of the response at frequencies lower than a certain limit, called the resonance frequency. The accuracy of the measurements is better for frequencies above this resonant frequency.

\section{Geophones selected for the measurement of pavement deflections}

To select appropriate geophones for pavement deflection measurements, several criteria have been considered:

- Appropriate sensitivity, to measure pavement deflections. Typical deflection levels in pavements are between $0.1 \mathrm{~mm}$ and $1 \mathrm{~mm}$.

- Low resonance frequency, in comparison with the frequency of deflection signals produced by road traffic (typically between 2 and $20 \mathrm{~Hz}$ ).

- Small size, to be able to embed the sensor in pavement layers.

- Good mechanical resistance and durability, to allow measurements in pavement layers for long periods (several years).

- $\quad$ Limited cost, to enable a widespread use of this type of instrumentation.

These criteria have led to select two types of geophones: geophone GEOSPACE GS11D with a sensitivity of $90 \mathrm{~V} / \mathrm{m} / \mathrm{s}$ (figure 1(a)) and Geophone Ion LF-24 (figure 1(b)), with a sensitivity of $15.2 \mathrm{~V} / \mathrm{m} / \mathrm{s}$.

Figure1(a)Geophone GS11D Figure1(b) Geophone ION
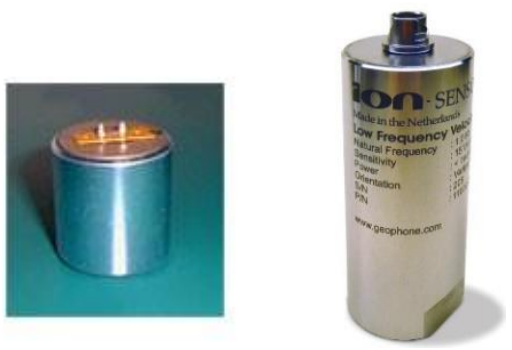

The frequency response curve (transfer function) of the sensors in figure 2 is established from the ratio of the signal delivered by the geophone and the signal supplied by the reference sensor. The sensitivity of the sensor is given by the "flat" part of the amplitude ratio of the response as a band of frequencies reported as a physical value. The flat part from 30 to $60 \mathrm{~Hz}$ 
represents the sensitivity of the sensors. For Geospace GS11D the sensitivity is considered to be $90 \mathrm{~V} / \mathrm{m} / \mathrm{s}$ and for the geophone ION the values is $15.25 \mathrm{~V} / \mathrm{m} / \mathrm{s}$

Figure 2: Transfer function of Geophones

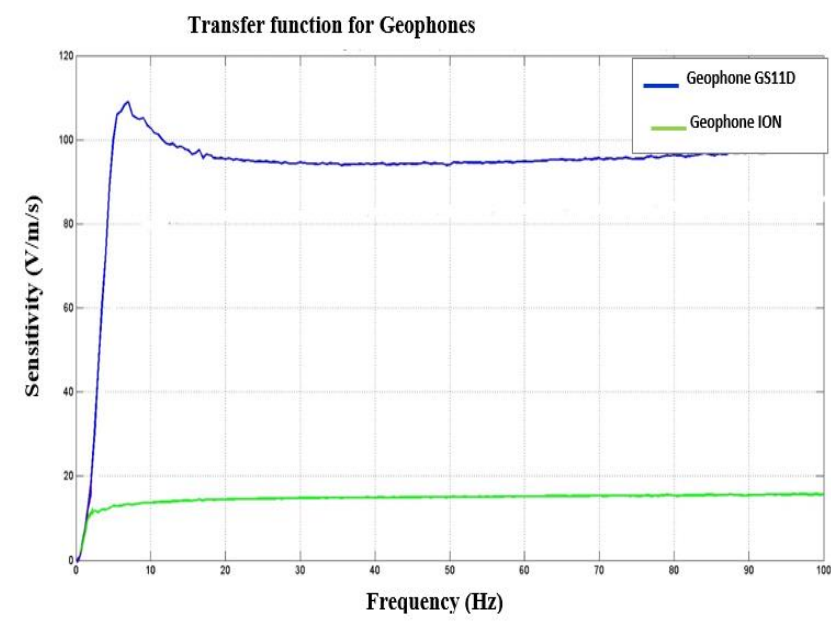

\section{Modelling of pavement deflections}

In France there are number of technical standards and documents that are followed for the structural designing of pavement. To define reference deflection signals for the laboratory tests, aimed at evaluating the response of the geophones, pavement calculations have been performed with the linear elastic software-Alize. This software is used to model the pavement structure which is multi-layer, elastic linear, semi infinite and continuous. The interfaces between theses layers can be bonded or un-bonded depending on the pavement materials used. It allows calculating the mechanical response of the pavement when subjected to static pressure loads in terms of longitudinal and horizontal strains, stresses and deflections. These calculations are based on the theory proposed by D. Burmister defining the solution for an axisymmetric problem for a single uniform circular pressure load (Remaud D 2017). It can also be used for back calculating pavement layer moduli as well, using the deflection basins. For our work, it was decided to perform calculations for a typical pavements structure, submitted to the loading of a 5 axles truck, with a total weight of 40 tons. The pavement structure considered is a classical flexible pavement for medium traffic (150truck/day) with a bituminous wearing course and granular base. The characteristics are defined in table1.

The deflection calculated with ALIZE for the flexible pavement, under the loading of a T2S3 (5-axles) truck, with a total load of 40 tons, and moving at a speed of $70 \mathrm{Km} / \mathrm{h}$, is presented on figure 3. Each peak of the signal represents the displacement produced by each wheel load as shown in the figure.
Table 1 Pavement model

$\begin{array}{lll}\text { Materials } & \begin{array}{l}\text { Elastic } \\ \text { Modulus(MPa) }\end{array} & \begin{array}{l}\text { Thickness } \\ \text { (cm) }\end{array}\end{array}$

\begin{tabular}{lcc}
\hline $\begin{array}{l}\text { Bituminous } \\
\text { mixture }\end{array}$ & 7000 & 11 \\
$\begin{array}{l}\text { Unbound granular } \\
\text { material }\end{array}$ & 200 & 30 \\
Subgrade & 80 & 250 \\
\hline
\end{tabular}

Figure 3: Calculated deflection of the flexible pavement under truck loading. The peaks correspond to the loads of the five axle's vehicle.

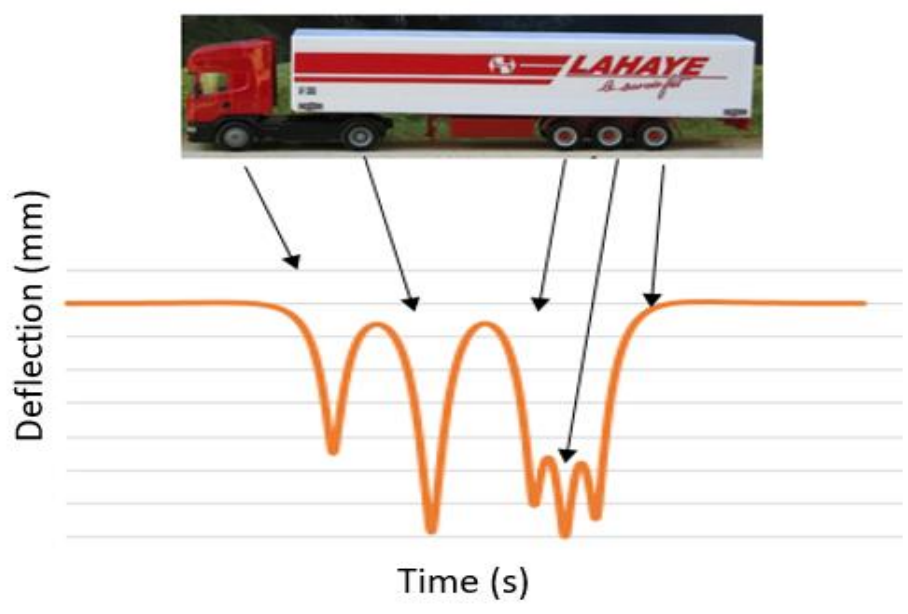

\section{Laboratory test protocol}

To evaluate the geophones, the following experimental protocol was defined:

The tests were performed using a vibrating table. This vibrating table uses a closed-loop servo hydraulic actuator, which applies the displacement to a horizontal platform, on which the transducers were installed. Four sensors can be tested at a time on the vibrating table, as the data acquisition system of the device has four channels. The displacements applied by the vibrating table were measured with a non-contact laser displacement sensor (Keyence, model LC2100, range $+/-8$ $\mathrm{mm}$ ) which was used as a reference sensor, to evaluate the response of the geophones as described in figure 4. This set up was used to measure the noise of the geophones, the frequency response as explained in section 3 and the response to pulse load and vehicle load. Different types of tests were successively performed to determine different characteristics of the geophones:

- The responses for different amplitudes and velocities

- The sensitivities and frequency response of the geophones 
- The intrinsic parameters that affect the response of the devices could also be studied

Figure 4: Vibrating table tests with various sensors

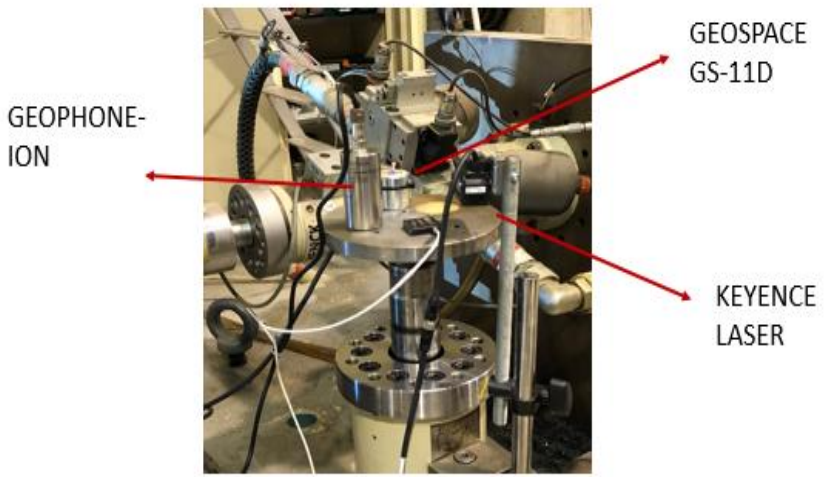

Two types of tests, characterising the response of the geophones in terms of vertical displacements were successively carried out on the vibrating table, as described in table 2 . The pulse signals simulate the response under a single axle load and hence the response shows a vertical displacement with a single pulse. The truck signals correspond to the signals generated using ALIZE (figure 3), corresponding to the deflections caused by a five axle truck. The shape of the calculated signals has been kept identical in all tests, but different signal amplitudes and frequencies, representative of different possible pavement loading conditions, have been applied in the tests. Figure 5(a) represent the deflections response from the vibrating table for a pulse amplitude of $0.1 \mathrm{~mm}$ and a speed of $70 \mathrm{Km} / \mathrm{h}$. Figure 5 (b) presents the corresponding displacement velocities in terms of truck signal measured by the geophones on the vibrating table at the amplitude of $0.1 \mathrm{~mm}$ and a speed of $70 \mathrm{Km} / \mathrm{h}$. However, these raw measurements given by the geophones need to be treated and converted in order to get the vertical displacements. Also these responses need to be processed to get an accurate response in terms of the reference displacements obtained from the reference sensor.

Table 2: Test settings

\section{Tests Details} $\begin{array}{ll} & \text { Reference signal: Keyence laser } \\ \text { Pulse Signal } & \text { Amplitude: } 0.1 \mathrm{~mm}, 0.3 \mathrm{~mm}, 1 \mathrm{~mm} \\ & \text { Speed: } 35 \mathrm{Km} / \mathrm{h} / 70 \mathrm{~km} / \mathrm{h}\end{array}$

Truck Signal

Reference Signal: Keyence Laser Amplitude: $0.1 \mathrm{~mm}, 0.3 \mathrm{~mm}, 1 \mathrm{~mm}$ Speed: $35 \mathrm{Km} / \mathrm{h}, 70 \mathrm{~km} / \mathrm{h}$
Figure 5 (a): Untreated raw pulse signal corresponding to the $70 \mathrm{~km} / \mathrm{h}$

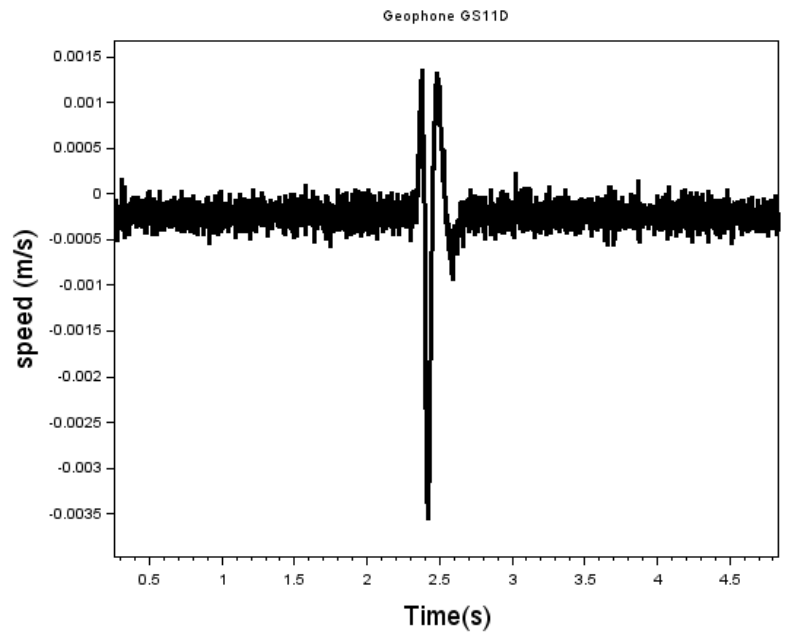

Figure 5(b): Untreated raw truck signal corresponding to the $70 \mathrm{~km} / \mathrm{h}$ load

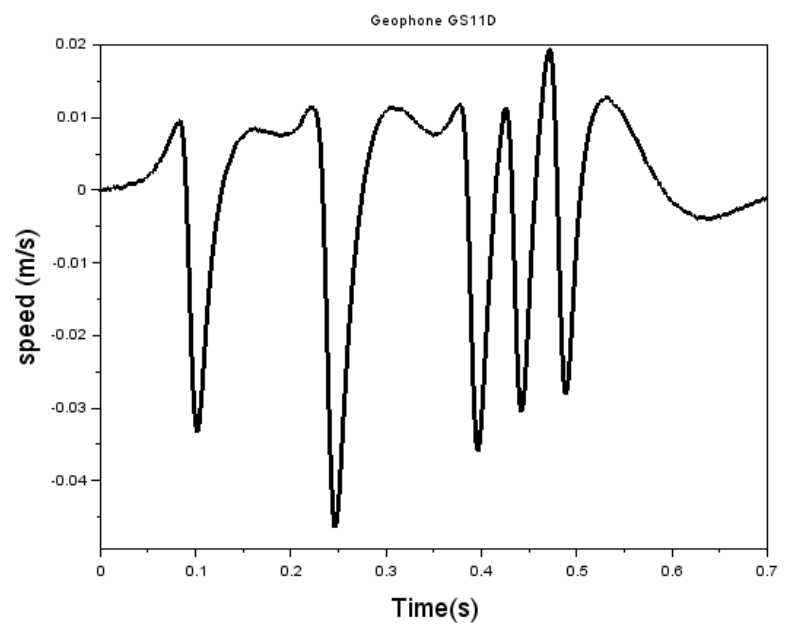

\section{Processing and improving the geophone Signal measurements}

The measurements of the geophones require some treatment in order to convert them into appropriate values of vertical displacements. The measurements need to be integrated from speed into vertical displacements (deflections). Figure 6 represents a raw geophone signal (measured displacement velocity), and the signal obtained after a simple integration (vertical displacement). It can be seen that after a simple integration, the displacement signal obtained does not correspond exactly to the reference signal applied to the vibrating table (signal calculated with ALIZE, shown on figure 3). The relative amplitude of the peaks is different from the reference signal, and the signal presents some positive parts, 
indicating an upward displacement, which is not realistic; only a downward (negative) displacement is applied to the geophone. After analysis, it seems that this distortion of the displacement signal may be due to two main reasons:

- The frequency response of the geophones, which tends to attenuate the low frequency components of the signal

- The integration process, which introduces a constant, and thus adds a continuous component to the signal. This may explain the unrealistic shape of the signal, with positive displacement values.

In addition, as can be seen on figure 5, the raw geophone signals has some noise, which can also lead to inaccurate results.

Figure 6: Example of raw and integrated signal of geophone GS11D, for a 5 axle truck loading.
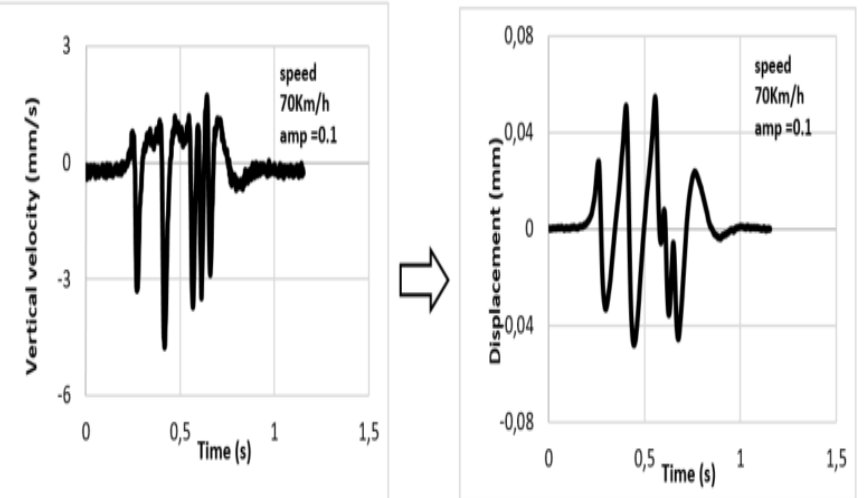

Initial vertical velocity without integration from geophone GS11D

Integrated signal in terms of displacement without processing

\subsection{Method to convert the signal into vertical displacement and improving the accuracy}

To improve the vertical displacements obtained from these geophones, various signal-processing methods have been tested and a methodology to correct the measurements has been developed. A signal correcting procedure has been applied to the measurements of the two types of geophones used (Geospace GS11D and Ion LF-24) but in the following figures explaining the treatment procedure, only the responses obtained with the geophone GS11D are shown. This methodology is described below:

\section{Step1: Filtering the signals with a high pass frequency filter}

The first step involves filtering of the raw signal and the important key is to filter the signal at an appropriate frequency in order to obtain a deflection value that matches the reference signal. As the noise of sensors effects mainly the lower

frequencies, a high pass filter allows to keep the essential part of the signal shown in figure 7 . However, it was observed that the cut off frequency of the filters needs to be adjusted according to the vehicle speed. At lower speeds, a lower cutoff frequency must be used than at higher speed. In this experiment, two speeds have been chosen, for the speed of $35 \mathrm{Km} / \mathrm{h}$ a cut-off frequency is of $10 \mathrm{~Hz}$ was found to give good results, while at $70 \mathrm{Km} / \mathrm{h}$ the cut off frequency had to be increased to $40 \mathrm{~Hz}$. At these, cut off frequencies the shape of the signal drastically improved.

Figure 7. Filtering of the geophone GS11D signal, with a high pass filter (frequency $40 \mathrm{~Hz}$ )

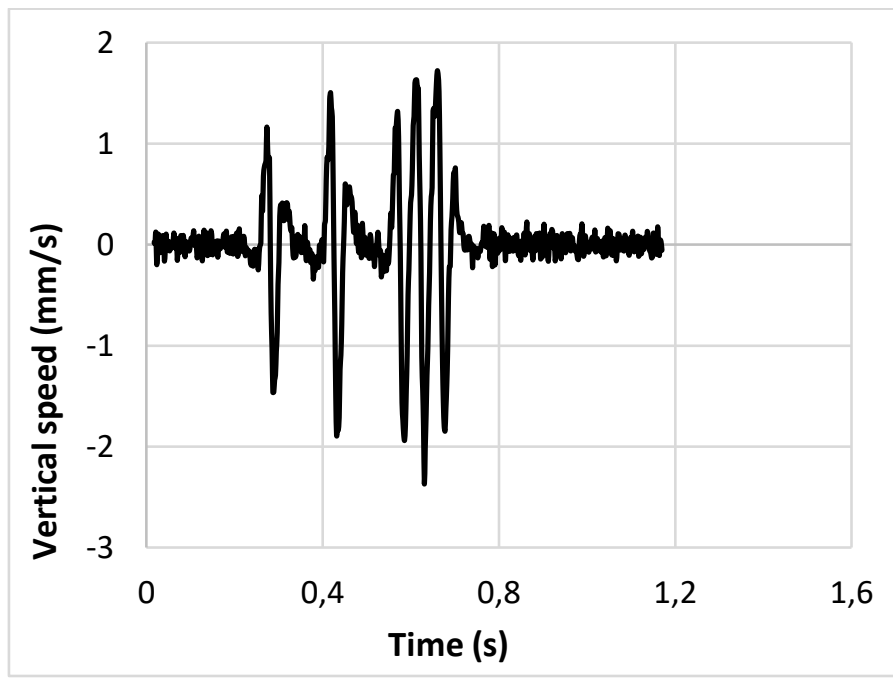

Step 2: Amplification of the signal with respect to the reference signal amplitude

When the cut off frequencies were kept lower then values indicated above and amplification was applied to the signal, it not only increased the required part of the signal but also the noise, hence the shape of the signal was deteriorated. At high frequency, filtering improved the overall shape of the signal but the down side of this is that the amplitude of the signal was reduced. When the final filtered signal was compared to the reference signal, the amplitude was much lower. For this reason, after filtering, the signal had to be amplified, to keep the initial amplitude as shown in the figure 8.

Step 3: Integration and detrending to remove the continuous part of the signal

After filtering, the signal is integrated to be converted into vertical displacement. As mentioned above, the integration step adds a continuous component to the signal. Hence, a detrending function was applied to suppress this continuous component. The signal after integration and the detrending is shown in the figure 9. 
Figure 8. Amplification of the geophone GS11D signal after filtering

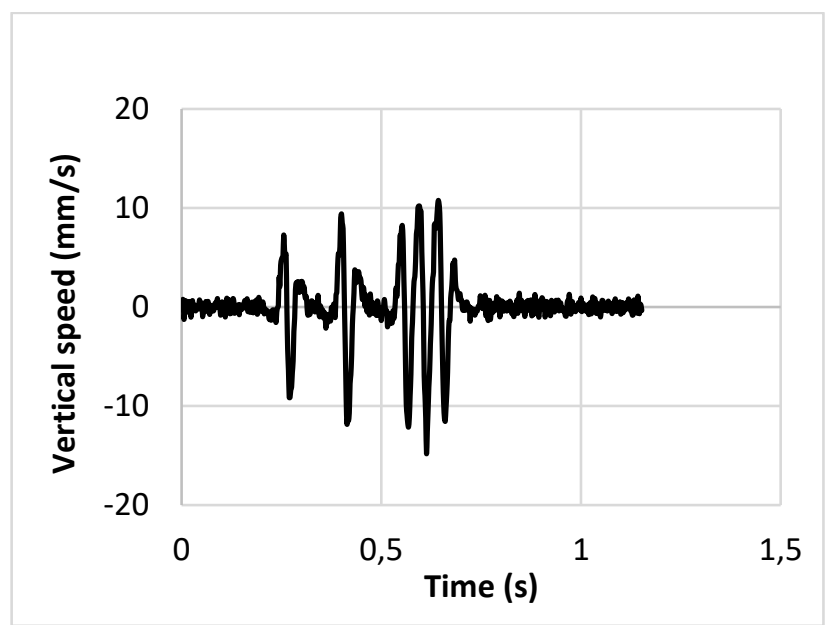

Figure 9. integration of the geophone GS11D signal

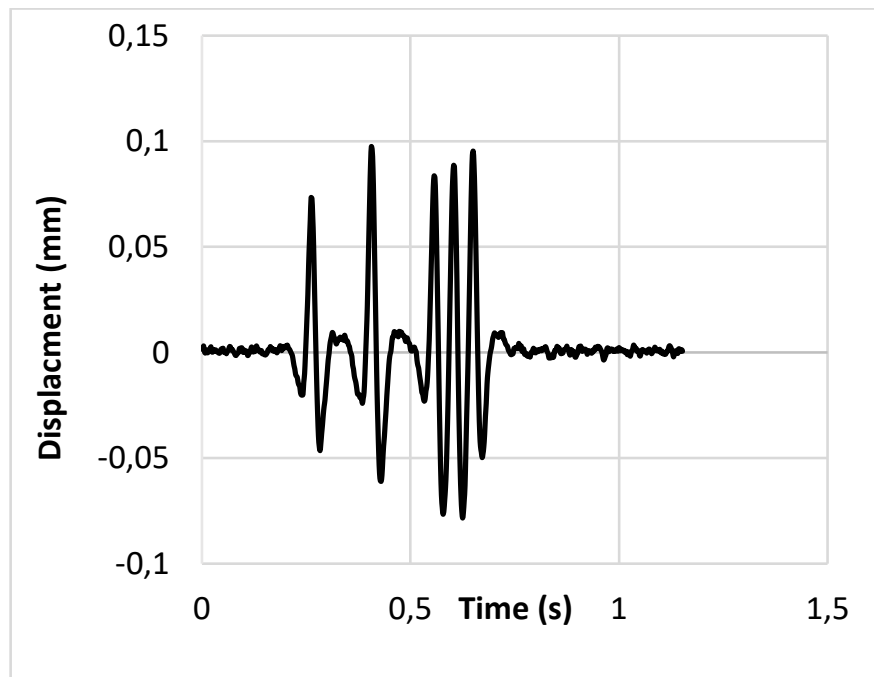

\section{Step 4: Application of the Hilbert transform}

Finally a Hilbert transform is applied to remove the oscillations in the signal and further enhancing the shape of the signal. The common application of the Hilbert transform is in the demodulation operation for extracting the envelop of the initial signal. It shifts the phase component of the signal by \pm 90 degrees so when the signal is combined with its Hilbert transform the periodical oscillations in the signal are removed and the upward lifts in the signal are shifted. This transform drastically improves the shape of the signal, and leads to a response close to the theoretical deflection under five-axle vehicle as evident in figure 10 .
Figure 10. Final signal of geophone GS11D after application of the Hilbert transform

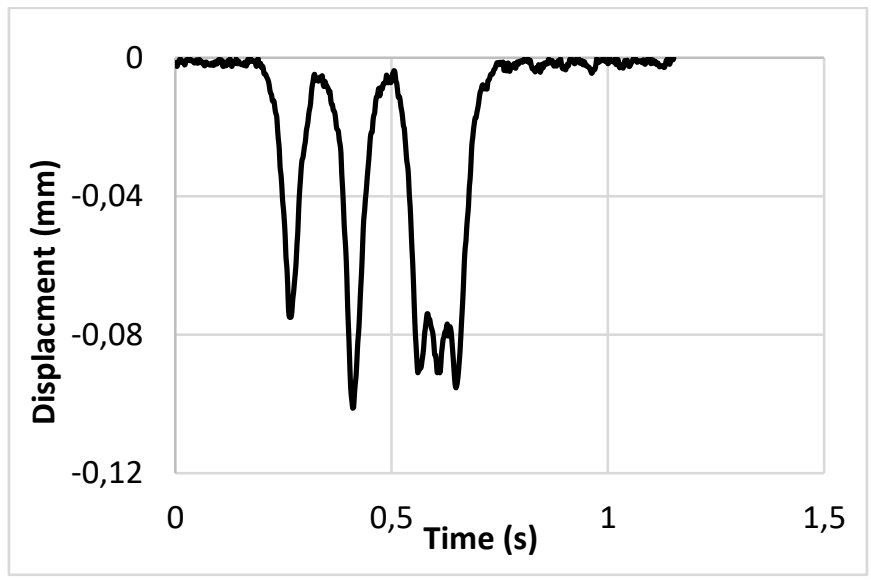

\subsection{Comparison of the treated geophone measurements with the reference laser sensor}

Finally, the proposed correction procedure has been applied to the geophone signals obtained at different speeds and amplitudes, and the results have been compared with the reference displacement values obtained with the Keyence Laser sensor. Globally, the procedure improves the geophone measurements very significantly and leads to a realistic signal shapes and displacement amplitudes, as described in the section below.

Figure 11: Example of comparison between the geophone signal obtained with and without the improved processing method
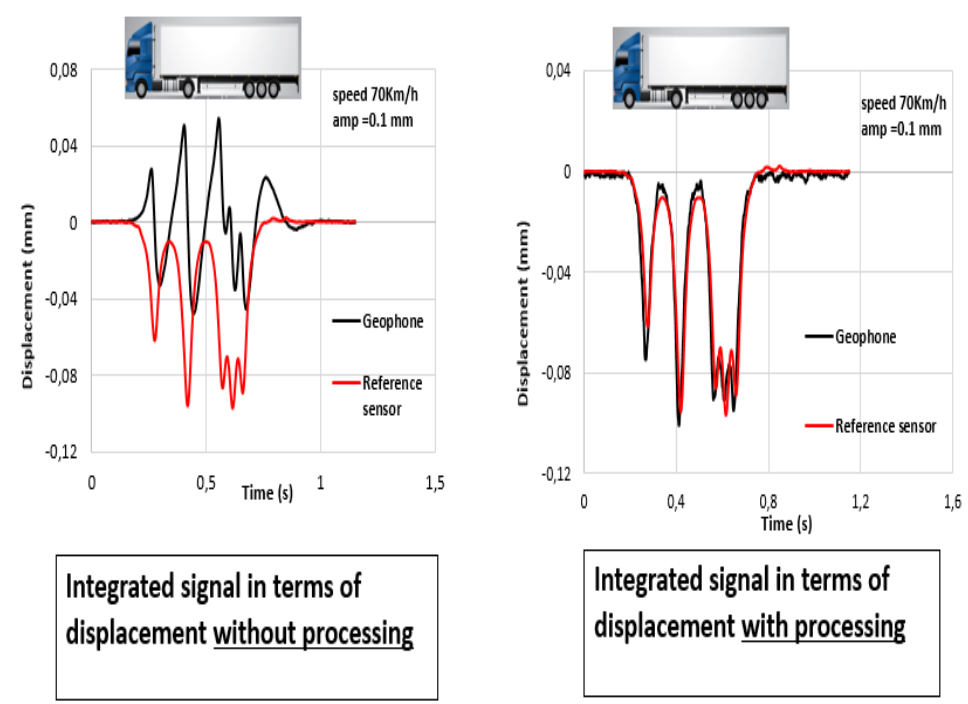


\section{Results}

This section presents examples of treated displacement measurements obtained with the geophone GS11D and the geophone ION LF-24 for different speeds and amplitudes. This processing is applied to the responses obtained from the tests as described in the table 2. The treated signals are compared with the response of the Keyence laser displacement sensor. It is important to note that the treatment procedure is the same for all the amplitudes and speeds. The only parameter which is different is the cut-off frequency of the high pass filter. It has been noted that as the speed increases, it is necessary to increase the cut off frequency of the filter. For the speed of $35 \mathrm{~km} / \mathrm{h}$, the suitable cut off frequency is $10 \mathrm{~Hz}$, and for $70 \mathrm{~km} / \mathrm{h}$ it needs to be increased to $40 \mathrm{~Hz}$. With these cut off frequencies, the optimal shape of signal is achieved, which is very close to the reference signal.

\subsection{Pulse signals}

The following results represent the processed pulse signal corresponding to a single axle load and with speed of $35 \mathrm{Km} / \mathrm{h}$ and $70 \mathrm{Km} / \mathrm{h}$ and with amplitudes of the vertical displacement of $0.1,0.3$ and $1 \mathrm{~mm}$. After applying the improved processing procedure, realistic displacements amplitudes were obtained for all the test conditions when compared to the reference sensor signal, slightly better results were obtained with GS11D geophone.

Figure 12: Deflection of $0.1 \mathrm{~mm}$ amplitude and speed $35 \mathrm{Km} / \mathrm{h}$

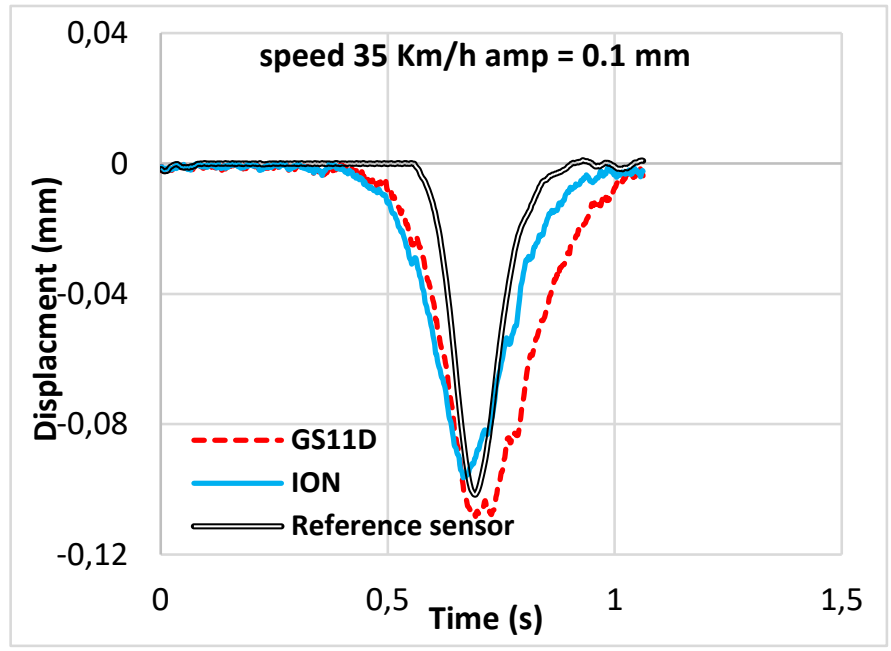

Figure 13: Deflection of $0.3 \mathrm{~mm}$ amplitude and speed $35 \mathrm{Km} / \mathrm{h}$

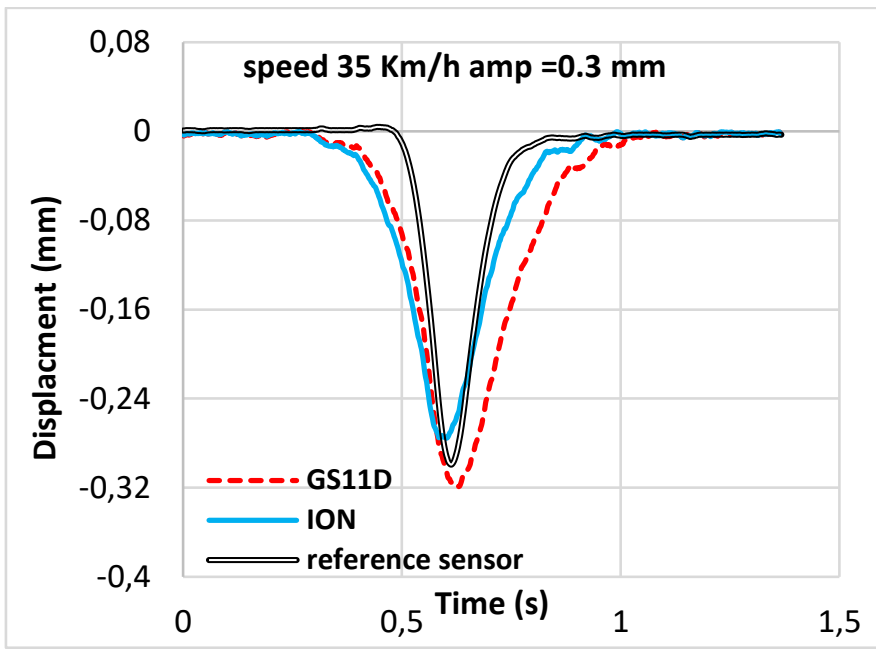

Figure 14: Deflection of $1 \mathrm{~mm}$ amplitude and speed $35 \mathrm{Km} / \mathrm{h}$

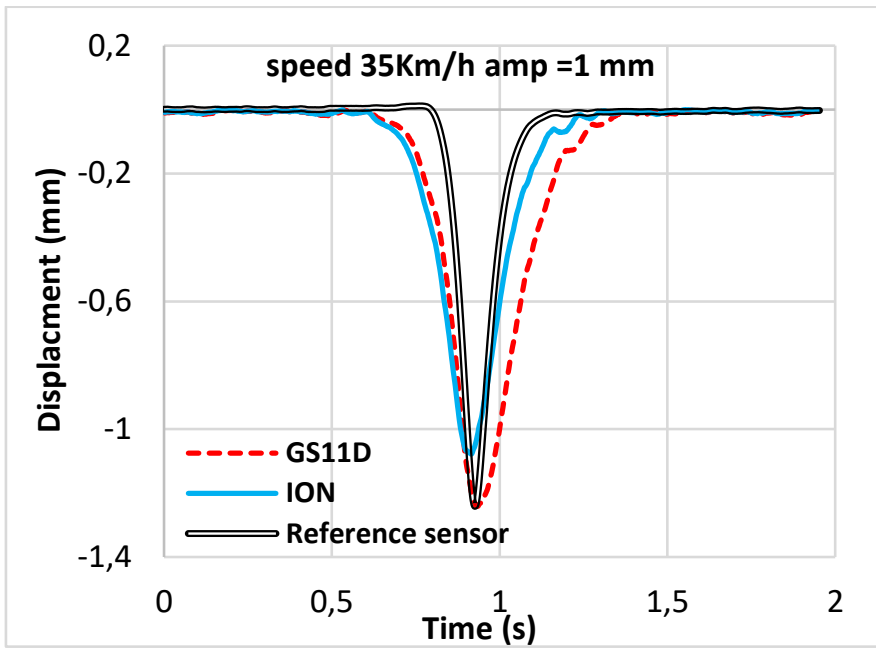


Figure 15: Deflection of $0.1 \mathrm{~mm}$ amplitude and speed $70 \mathrm{Km} / \mathrm{h}$

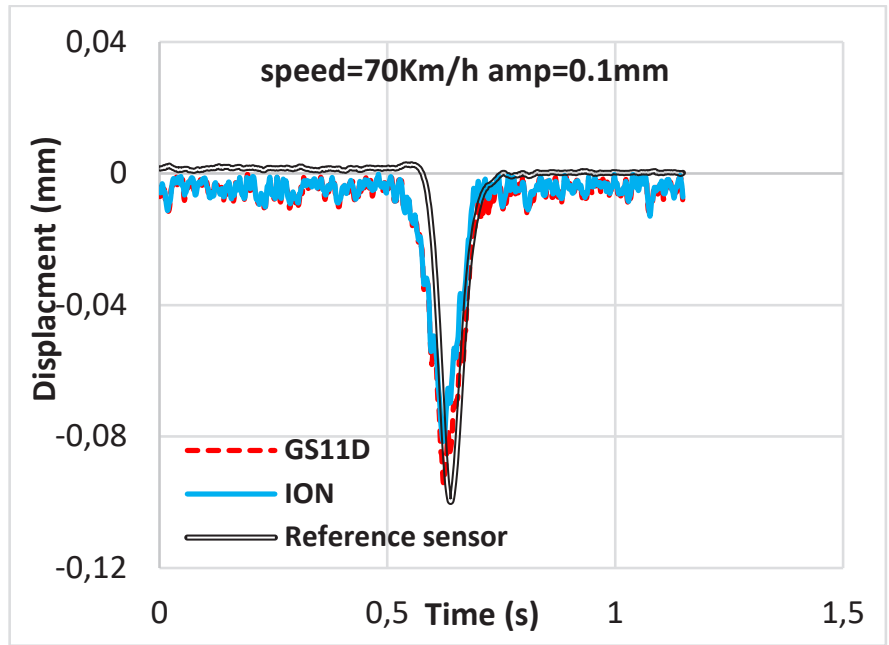

Figure 16: Deflection of $0.3 \mathrm{~mm}$ amplitude and speed $70 \mathrm{Km} / \mathrm{h}$

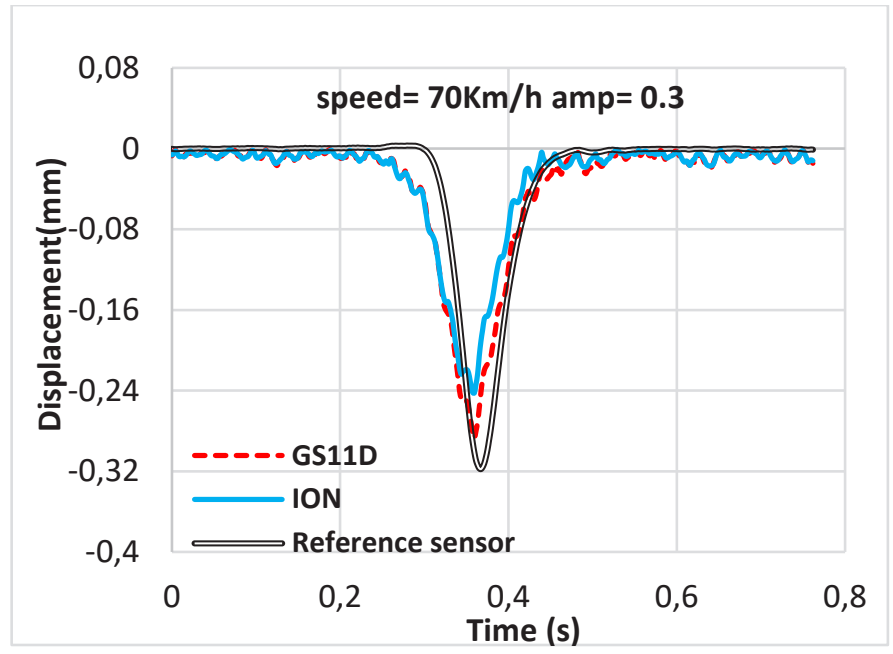

Figure 17: Deflection of $1 \mathrm{~mm}$ amplitude and speed $70 \mathrm{Km} / \mathrm{h}$

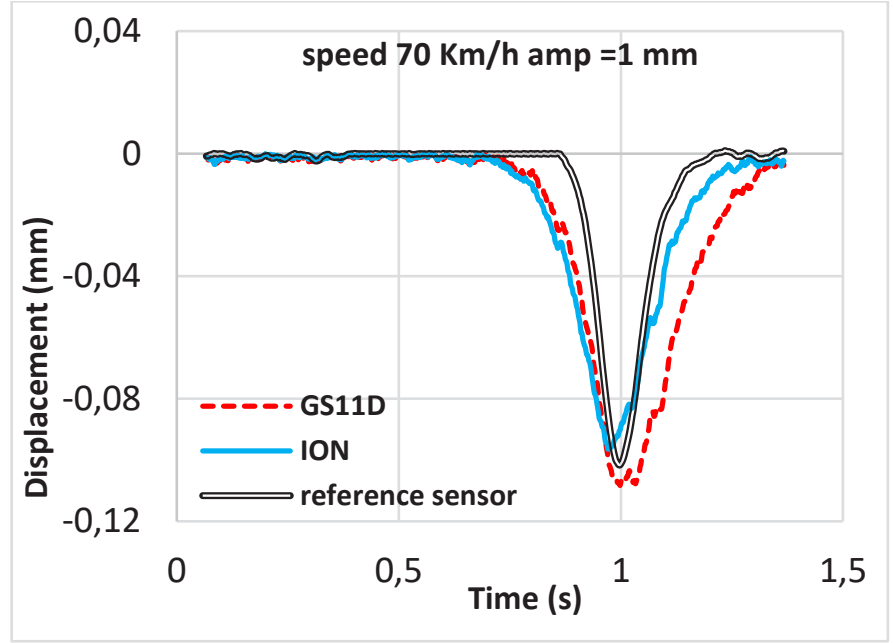

\subsection{The truck signal}

The following results represent the truck signal corresponding the five axle's loads, with the speeds of $35 \mathrm{Km} / \mathrm{h}$ and $70 \mathrm{Km} / \mathrm{h}$ and with the vertical displacement amplitude of $0.1,0.3$ and 1 $\mathrm{mm}$. Similar to the pulse signal results. The processed geophone signal present amplitudes and shapes in good agreement with the reference signal and again the geophone GS11D predicts slightly better signal amplitudes.

Figure 18: Deflection of $0.1 \mathrm{~mm}$ amplitude and speed $35 \mathrm{Km} / \mathrm{h}$

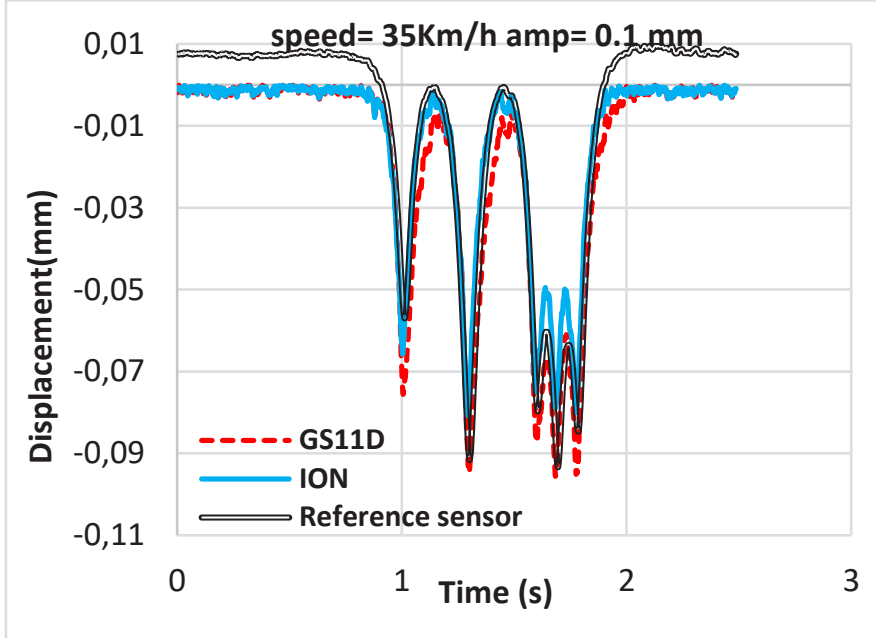

Figure 19: Deflection of $0.3 \mathrm{~mm}$ amplitude and speed $35 \mathrm{Km} / \mathrm{h}$

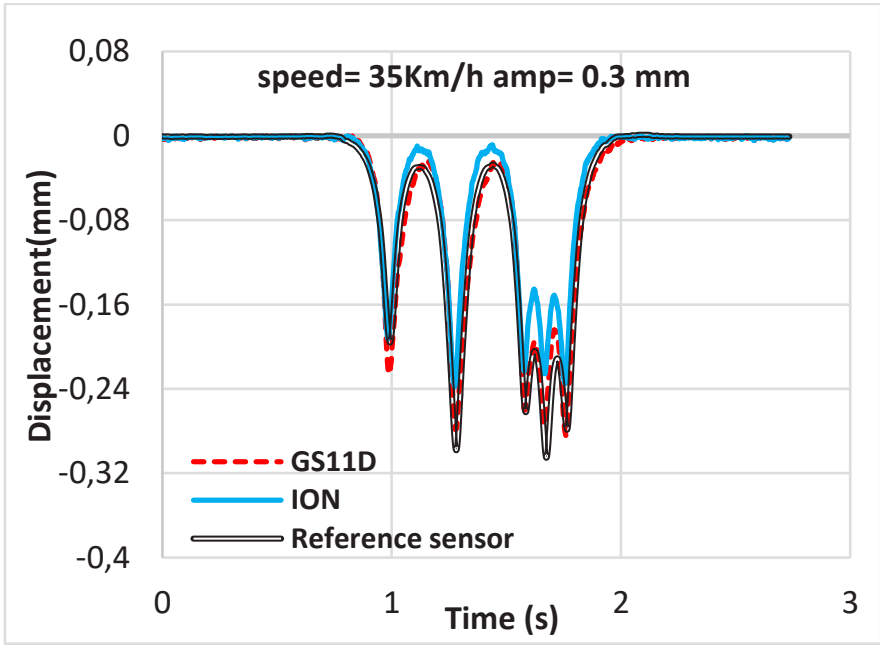


Figure 20: Deflection of $1 \mathrm{~mm}$ amplitude and speed $35 \mathrm{Km} / \mathrm{h}$

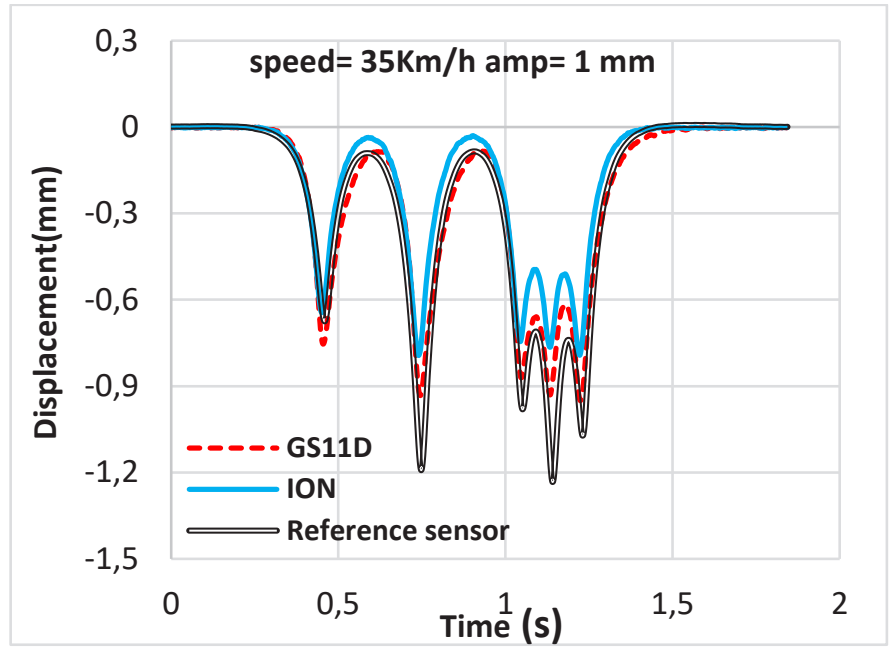

Figure 21: Deflection of $0.1 \mathrm{~mm}$ amplitude and speed $70 \mathrm{Km} / \mathrm{h}$

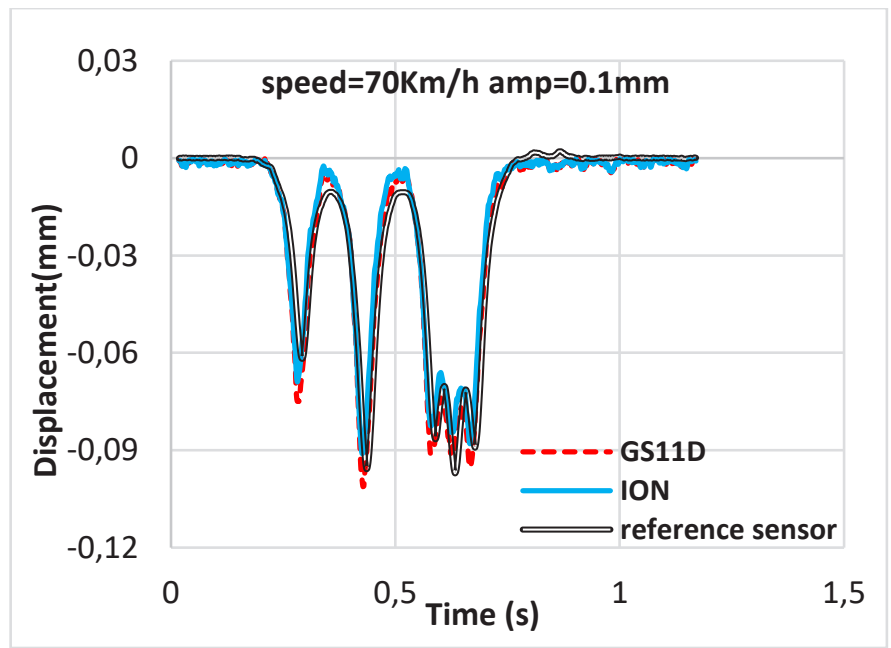

Figure 22: Deflection of $0.3 \mathrm{~mm}$ amplitude and speed $70 \mathrm{Km} / \mathrm{h}$

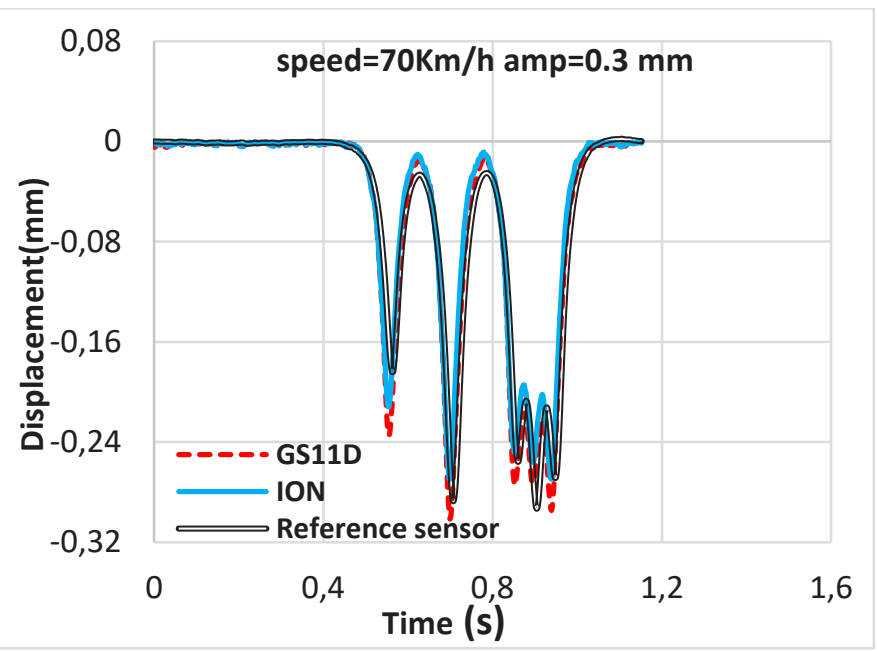

Figure 23: Deflection of $1 \mathrm{~mm}$ amplitude and speed $70 \mathrm{Km} / \mathrm{h}$

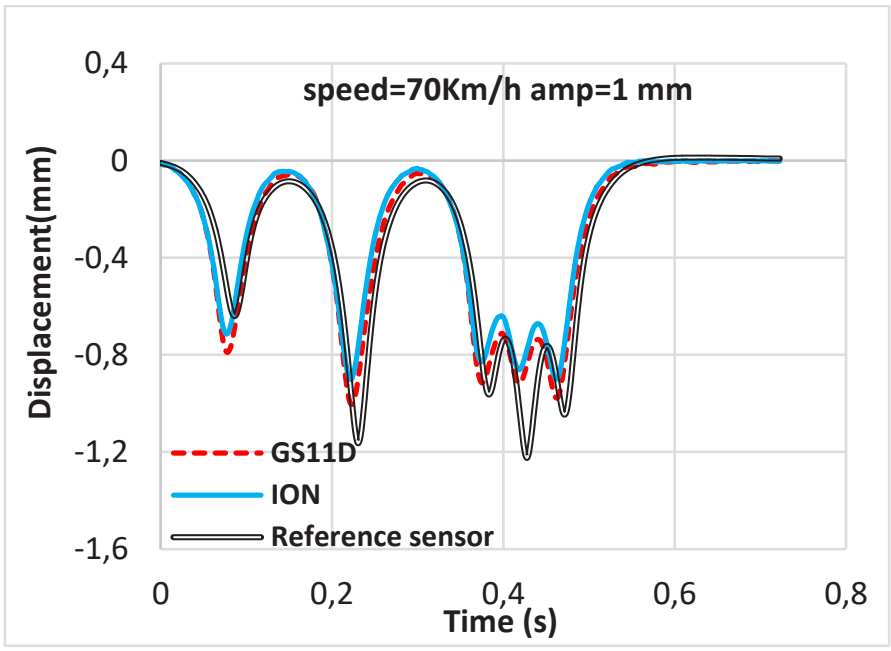

\section{Conclusion}

Geophones are sensors developed for the measurement of displacement velocities. They are used in various applications, such as detection of ground motions, for seismic applications. The objective of this study was to evaluate the possibility to use geophones for pavement instrumentation, to monitor pavement deflections under vehicle traffic. After defining the requirements for such measurements, two types of geophones have been selected for the study: a geophone Geospace GS11D and a geophone Ion LF-24. Before testing the geophones in real conditions in a pavement, a laboratory study was performed, to evaluate the response of the geophones to simulated pavement deflection signals, generated by a hydraulic vibrating table.

The geophones were submitted to sinusoidal pulse signals, and to signals reproducing the deflection under a five axle truck. These signals were applied with different amplitudes, and different frequencies, corresponding to the range of conditions encountered in real pavements.

The first results indicated that a simple integration of the geophone signals, to convert the measured displacement velocity into displacement is not sufficient to obtain accurate displacement values. Different treatments of the signals have been tested to improve the response, and a correction procedure has been proposed. This procedure includes the following steps: noise filtering, amplification, integration of the signal, detrending, and application of a Hilbert transform. The treated signals have been compared with reference displacement measurements, carried out with a reference laser displacement sensor, and it has been shown that after treatment, realistic deflection values can be obtained for typical pavement loading conditions. 
These results confirm the possibility of using embedded geophones for the measurement of pavement deflections. Geophones present interesting characteristics for this type of measurements, due to their high sensitivity, good robustness, and relatively low cost. Following these results, it is planned to validate the results obtained in the laboratory by a full scale experiment, on a real pavement section. Work is also under way to fully automate the signal treatment procedure, and implement it in the field data acquisition system, to obtain deflection measurements in real time, and avoid the posttreatment of the results.

The final objective is to develop a low cost and fully automated system for in situ measurement of pavement deflections, that could be deployed on road networks, for pavement monitoring. Such deflection measurements can then be used for backcalculating pavement layer properties, and monitoring their evolution with time.

\section{References:}

\section{Journal Articles}

Lajnef N et al, "Toward an Integrated Smart Sensing System and Data Interpretation Techniques for Pavement Fatigue Monitoring", Computer-Aided Civil and Infrastructure Engineering Volume26, Issue7, October 2011, Pages 513-523

Arraigada $\mathrm{M}$ et al. Evaluation of accelerometers to determine pavement deflection under traffic loads, journal materials and structures(2009) 42/ 779-790

Levenberg E, Inferring Pavement Properties using an Embedded Accelerometer, international journal of transportation and technology, vol 1, Pg-229-246

Alavi. AH et al, "Continuous health monitoring of pavement systems using smart sensing technology", Construction and Building Materials, Volume 114, 1 July 2016, Pages 719-736

Liu. $\mathrm{P}$ et al, "Measurement and evaluation on deterioration of asphalt pavements by Geophones", in: Measurement, volume $109,(2017), 223-232$.

Duong N.S et al, Monitoring of pavements using the geophones, 2018, International journal of pavements Engineering.

\section{Reports}

Tholen $\mathrm{O}$ et al, Comparison of FWD with other deflection testing devices. Transportation research record 1007, TRB Washington, DC: National Research Council; 1985 p. 20-26.
Clark R. et al, Calculating Pavement Deflections with Velocity Transducers, Proceedings, Transportation Research record 1293,TRB, 1991.

Nazarian, S. et al, "Determination of Deflection of Pavement Systems Using Velocity Transducers," Proceedings, Transportation Research Record 1227,TRB, pp. No. 147-158, 1989.

Remaud D et al, Alize-LCPC software Analysis and structural design of pavement structures, RGRA hors série report, December 2017 